\title{
AUTOESTIMA Y DESESPERANZA EN ADOLESCENTES DE UNA INSTITUCIÓN EDUCATIVA DEL QUINDÍO
}

\section{ESTEEM AND HOPELESSNESS IN ADOLESCENTS IN AN EDUCATIONAL INSTITUTION OF QUINDÍO}

\section{TÍTULO CORTO: AUTOESTIMA Y DESESPERANZA EN ADOLESCENTES}

\section{José Alonso Andrade-Salazar' ${ }^{1}$, Lowell Duffay-Pretel ${ }^{2}$, Paola Andrea Ortega-Maya ${ }^{3}$, Elberney Ramirez-Avilés ${ }^{4}$, Jorge Erik Carvajal-Valencia ${ }^{5}$}

Recibido en mayo 05 de 2016

Aceptado en octubre 19 de 2016

Publicado en línea en mayo 15 de 2017

\section{Resumen}

Esta es una investigación empírico-analítica de tipo correlacional cuya intención es hallar la relación entre niveles de autoestima y desesperanza en 224 adolescentes de los grados $9^{\circ}, 10^{\circ}$ y $11^{\circ}$ de bachillerato de una institución educativa pública del departamento del Quindío. Se aplicó la escala de Desesperanza de Beck y el inventario de autoestima de Rosenberg. Se encontró que el Riesgo de Suicidio o grado de Desesperanza "ninguno o mínimo" fue del $59,4 \%$, el riesgo leve $26,8 \%$, moderado $12,9 \%$ y alto $0,9 \%$. El riesgo mínimo en mujeres fue del $33,5 \%$ y alto del 0,9\%, mientras en los hombres prevalece el riesgo mínimo $29,5 \%$, leve $17 \%$ y moderado $7,1 \%$. La desesperanza se incrementa en los adolescentes por factores motivacionales y cognitivos que dan forma a la ideación suicida y elevan los riesgos de suicidio. La autoestima baja no es un factor consistente en la ideación suicida.

Palabras clave: Adolescencia; autoestima; pesimismo; depresión; suicidio.

\section{Abstract}

This is an empirical-analytic correlational research whose intent is to find the relationship between levels of self-esteem and hopelessness in adolescents in an educational institution of Quindío-Colombia. The Beck Hopelessness Scale

1. Psicólogo. Docente investigador. Investigador del grupo interdisciplinario para el desarrollo y la acción dialógica (GIDPAD) y del grupo Estudios clínicos y sociales en psicología, Facultad de psicología. Universidad de San Buenaventura. Armenia, Colombia Correo: jose.andrade@usbmed.edu.co - http://orcid.org/0000-00017916-7409

2. Estudiante de X semestre del programa de Psicología. Universidad de San Buenaventura. Armenia, Colombia. Correo: lowellduffay@hotmail.com - http://orcid. org/0000-0002-8127-8412

3. Estudiante de X semestre del programa de Psicología. Universidad de San Buenaventura. Armenia, Colombia.. Correo: paolita1800@hotmail.com - http://orcid. org/0000-0001-7373-8602

4. Estudiante de X semestre del programa de Psicología. Universidad de San Buenaventura. Armenia, Colombia. Correo: avirael@hotmail.com - http://orcid.org/00000002-7608-5763

5. Estudiante de X semestre del programa de Psicología. Universidad de San Buenaventura, Armenia, Colombia. Correo: carvajalps.92@hotmail.com - http://orcid. org/0000-0001-6360-8353 
and the Self-Esteem Scale Rosenberg were applied. It was found that the risk of suicide or grade of hopelessness "none or minimal" is $59.4 \%$, "mild" $26.8 \%$, "moderate" $12.9 \%$ and "high" $0.9 \%$. The type of suicide risk is minimal in women $33.5 \%$ and high $0.9 \%$, while in the men is minimal $29.5 \%$, mild $17 \%$, and moderate $7.1 \%$. Expressing as conclusion that hopelessness is increase for motivational and cognitive factors that shape suicidal ideation and raise the risk of suicide. Low self-esteem is not a consistent factor in suicidal ideation.

Keywords: Adolescence; Self-esteem; Pessimism; Depression; Suicide.

\section{INTRODUCCIÓN}

$\mathrm{E}^{\mathrm{l}}$ suicidio es un problema de salud pública que afecta a diversos grupos etarios y en todos los casos deteriora la calidad y expectativa de vida de las familias que lo experimentan; para la sociedad en general existe una tendencia a mirar con terror y asombro el fenómeno cuando se presenta a edades tempranas ${ }^{1-4}$, motivo por el cual existen en los adultos resistencias e incluso negación ante la posibilidad de su emergencia ${ }^{2,5,6}$. Una definición articulada del suicidio puede pensarse a partir del reconocimiento e interrelación entre las conductas del espectro suicida, de modo que el comportamiento suicida se piense a modo de continuum configurado por la interrelación entre factores que van desde las primeras expresiones auto-líticas, hasta el suicidio consumado ${ }^{7}$. Los niños, niñas y adolescentes son sensibles a los cambios psicosociales, especialmente si en su familia y entorno se genera un estado permanente de vulnerabilidad ${ }^{8-11}$, aspectos que pueden aminorarse si se promueven espacios educativos protectores, políticas públicas y acciones preventivas del suicidio en niños, niñas, adolescentes y jóvenes ${ }^{12-16}$. De allí que el objetivo del presente estudio sea encontrar la posible relación entre autoestima y desesperanza (riesgo suicida) en estudiantes de bachillerato de los grados $9^{\circ}, 10^{\circ}$ y $11^{\circ}$, ya que su edad representa un periodo de intensos cambios y contradicciones que aumentan sus factores de riesgo vital $^{16}$.

180

En Colombia según el Instituto de Medicina Legal y Ciencias Forenses (Forensis) el suicidio de niños, niñas $\mathrm{y}$ adolescentes en edades entre 5-19 años, tiene una tendencia decreciente así: para el 2010 se presentaron 333 casos, y se redujo en el 2011 a 301, luego se incrementó en 2012 a 325, y en el 2013 hubo 300 casos disminuyendo a 293 en el 2014 siendo la cifra más baja en los últimos cinco (5) años. En el departamento del
Quindío se presentaron 47 suicidios en 2010 y aumentó a 55 en el 2011, luego disminuyó en los años siguientes. En el 2012 hubo 39 suicidios, 33 en el 2013, y 32 en el 2014; la tendencia fue de 8 suicidios de hombres por una mujer ${ }^{2-4}$, consolidándose la esfera nacional como el tercer departamento con mayor tasa por densidad poblacional (5,9 x 100.000 habitantes). Los informes de medicina legal no detallan el número de niños, niñas y adolescentes que se han quitado la vida por departamento y por ello no se cuenta con dicha información; sin embargo en su boletín epidemiológico reitera que según la OMS, 100.000 adolescentes se suicidan cada año en el mundo y que por cada evento consumado existen entre 10 y 20 intentos de suicidio ${ }^{17}$. Colombia presenta tasas menores a Chile, Argentina, Norteamérica y Europa, sin embargo, el suicidio es un problema de salud que entre menores de 5-18 años paso de 1,57 en el 2008 a 1,75 en 2012 según lo informa Forensis ${ }^{17,18}$.

El suicidio es la acción auto-provocada que tiene como corolario la muerte, e implica un elevado grado de decisión y planeación ${ }^{19,20}$. La conducta autodestructiva prevalece en la población joven y adulta, no obstante, en niños, niñas y adolescentes cuya capacidad de elección está mediatizada por la calidad de las interacciones con adultos, el suicidio puede asociarse "a trastornos psicopatológicos del espectro de la impulsividad o familias muy desorganizadas, lo cual conlleva un riesgo para la vida del niño sin conciencia e intención del acto que realiza" . Para Durkheim el suicidio es todo acto de muerte que emerge directa o indirectamente como efecto de un acto intencional en el que la persona sabe los posibles resultados de su conducta ${ }^{21}$; Durkheim plantea tres tipologías de suicidio: a) suicidio egoísta, o acto dado por el individuo cuando pierde la identidad respecto a su medio social, a manera de auto sacrificio ${ }^{5}$; b) suicidio altruista, presente en sociedades rígidamente estructuradas, donde el individuo posee un sentido 
grupal que influye en que su sacrificio sea una especie de exigencia moral, y c) suicidio anómico, producto de la falla o ruptura de los valores sociales que lleva al individuo a una desorientación, y tenga un sentimiento de vacío donde no encuentra un verdadero significado a la vida ${ }^{21,22}$.

Para Durkheim, el suicidio resulta de la ruptura de códigos morales y convenciones sociales, emergiendo como respuesta ante la elevada vulnerabilidad del sistema social ${ }^{22}$. Paralelamente el modelo arquitectónico de Mack ${ }^{23}$ explica dicha conducta a través de varios factores: Macrocosmos, vulnerabilidad biológica, experiencias tempranas, organización de la personalidad, relaciones del individuo, psicopatología, ontogenia y circunstancias vitales. El Macrocosmos deriva de la influencia ejercida por el sistema socio-político, cultural, educativo y económico; la vulnerabilidad biológica envuelve diversos factores genéticos asociados; las experiencias tempranas revelan riesgos vitales en la infancia; la organización de la personalidad se asocia al desarrollo Yoico y la autoestima; las relaciones del individuo involucran duelos, alianzas familiares y sociales, identificaciones y lazos afectivos; la psicopatología vincula al suicidio a trastornos mentales, consumo de alcohol y otras patologías; que la ontogenia conlleva la relación desarrollo-muerte, y las circunstancias vitales el tipo de interacciones socio-familiares.

Asimismo el modelo cúbico de Shneidman en $1976^{16,19}$ señala cuatro constructos relacionados con el comportamiento suicida: hostilidad, perturbación, constricción y cese, además de tres condiciones que suscitan el suicidio: a) la hostilidad hacia sí mismo que aumenta en los momentos previos a quitarse la vida; $b$ ) estados emocionales negativos y la forma en que influyen en las conductas; c) las características cognitivas dirigidas a la imposibilidad de percibir claramente la realidad de los problemas. En los adolescentes, estos indicadores se relacionan también dificultades para recibir y reconocer el apoyo social e intrafamiliar requerido para resolver los conflictos ${ }^{24}$. Los adolescentes presentan una elevada vulnerabilidad frente a los accidentes, violencia, suicidios, embarazos prematuros, consumo de sustancias, hábitos alimenticios y enfermedades de transmisión sexual según refiere la Organización Mundial de la Saludd ${ }^{25}$. La adolescencia es una etapa de cambios y fluctuaciones emocionales que deben propiciar el proceso hacia la madurez psicológica y social, generar identidad, proyecto vital y habilidades de afrontamiento $0^{1,23,26-28}$, lo que implica ruptura y a la vez dependencia de las figuras paternas ${ }^{2,20}$ dado que la influencia de los padres es crucial para el aprendizaje y afianzamiento de factores protectores, así como también de factores de riesgo relacionados a conductas autodestructivas ${ }^{29,30}$.

Existen muchos elementos precipitantes del suicidio que afectan la salud mental y física de los niños, niñas y adolescentes tales como: conflictos intrafamiliares, problemas escolares, influencia de grupos de pares peligrosos, crecer en entorno violento y/o vulnerable, crecer sin un proyecto de vida, además de factores psicológicos como depresión, angustia, trastornos del humor y problemas de autoestima, relacionados con un acontecimiento afectivo que se experimenta como una sensación o emoción intensa y positiva, que se afecta de acuerdo a la percepción del éxito, fracaso y la proyección del individuo9. En la autoestima de los adolescentes el sí mismo "self" se constituye a razón de cuatro concepciones: autoestima como objeto de atención para el sujeto, autoestima vivida como real e ideal, como respuestas psicológicas del self, y a modo de componente de la personalidad del sí mismo ${ }^{10}$ capaz de integrar recursos cognitivos y emocionales de acuerdo a las circunstancias experimentadas. La baja autoestima puede tornarse en factor de riesgo significativo cuando el entorno afectivo y socio familiar no sostiene y protege positivamente a la persona ${ }^{9,27,28,30-32}$.

\section{MATERIALES Y MÉTODOS}

\section{Diseño}

Esta es una investigación empírico-analítica con un diseño descriptivo-correlacional. La investigación empíricoanalítica es de tipo fáctica y en ella los hechos investigados realmente acontecen ${ }^{33}$.

\section{Población}

La muestra de esta investigación fueron 224 adolescentes que cursaron los grados $9^{\circ}, 10^{\circ}$ y $11^{\circ}$. El estudio se llevó a cabo en una institución educativa pública. El muestreo fue de tipo aleatorio simple. Se trabajó con estudiantes entre 13 y 19 años de edad.

\section{Instrumentos}

Se implementó una ficha de datos socio-demográfica, la escala de Desesperanza de Beck, Beck et al ${ }^{34}$ en su versión castellana, y la escala de autoestima de Rosenberg ${ }^{31}$, validada por Rojas-Barahona et al. ${ }^{28}$. La escala cuenta con una consistencia interna de $0,76-0,87$, y fiabilidad de 0,80 . La escala de Beck es un instrumento auto aplicado 
diseñado para evaluar desde una mirada subjetiva las experiencias personales acerca del futuro y bienestar propios, además de la habilidad para sobrellevar la adversidad. Las puntuaciones obtenidas en desesperanza son un predictor útil de posible suicidio. Cuenta con veinte (20) ítems que se responden como verdadero o falso. Las respuestas verdaderas en los ítems $2,4,7,9,11,12,14$, $16,17,18$ y 20 valen un punto y las falsas en esos ítems 0 puntos. La puntuación total se logra al sumar todos los ítems y puede variar entre 0-20 puntos. Las respuestas falsas en los ítems $1,3,5,6,8,10,13,15$ y 19 valen un 1 punto y las verdadero en dichos ítems, 0 puntos. Los puntos de corte son: 0-8: riesgo bajo de cometer suicidio, 0-3: ninguno o mínimo, 4-8: leve-; 9-20: riesgo alto, 9-14: moderado y 15-20: alto. El factor afectivo se consigue sumando las respuestas de los ítems 1, 6, 13, 15 y 19 (va de 0 a 5); el factor motivacional de los ítems $2,3,9,11$, $12,16,17$ y 20 (va de 0 a 8 ); el factor cognitivo de la suma de los ítems 4, 7, 8, 14 y 18 (va de 0 a 5). La escala de autoestima de Rosenberg es una escala auto aplicada con la que se busca evaluar los sentimientos de satisfacción e insatisfacción respecto a sí mismo; tiene diez ítems de los cuales cinco se expresan positivamente y cinco de forma negativa. La consistencia interna de la escala validada para Colombia es de 0,61-0,63.

\section{Procedimiento}

La investigación implicó cinco fases: 1) Diseño de la investigación y verificación de condiciones bioéticas de la investigación, la cual implicó la aprobación del consentimiento informado por parte del comité de bioética de la universidad; 2) Revisión teórica y del estado de arte y consolidación de la información a través de rejillas de consolidación de información y fichas de recolección bibliográfica; 3) Recolección de datos: aplicación de instrumentos, y análisis de la información a través del SPSS ver. 20.0; para este análisis se utilizaron medidas de tendencia central y el coeficiente de Pearson para definir las correlaciones entre variables; 4) Informe de resultados de investigación; 5) Socialización de resultados a la comunidad académica y a las instituciones educativas que otorgaron los permisos para aplicar las pruebas.

\section{Declaración sobre aspectos éticos}

Partiendo de las consideraciones éticas señaladas en la Declaración de Helsinki esta investigación tuvo un bajo riesgo, y para llevarla a cabo se solicitó el aval del consentimiento informado al comité de Bioética de la Universidad de San Buenaventura, Medellín, el cual fue firmado por los participantes del estudio previo a la aplicación de los instrumentos. Se indicó a las personas que los resultados tienen un uso investigativo-académico, además de la confidencialidad de la información recopilada, el respeto a su dignidad y la protección de sus derechos y bienestar.

\section{RESULTADOS}

De la investigación participaron 224 estudiantes (49,1\% mujeres; 50,9\% hombres) de edades entre 13-15 años $(41,1 \%), 16-18$ años $(57,1 \%)$, y 19 años $(1,1 \%)$. En cuanto a la división socioeconómica (se consideró que a mayor estrato mayor poder adquisitivo - va de cero a seis) el $20,1 \%$ era de estrato dos (2) mientras el $33 \%$ fue de estrato tres (3); el 36,6\% cursa noveno, el 30,4 \% décimo y el $33 \%$ onceavo grado; la creencia religiosa prevalente fue católica $(56,7 \%)$ seguida de la cristiana $(21,4 \%)$. El $15,6 \%$ es hijo único, el 31,1\% afirma haber estado triste la mayor parte del día durante el último mes, y el 11,6 \% considera que lo castigan demasiado y sin razón. El 15,6 $\%$ ha presenciado violencia entre sus padres, el 7,1\% tiene uno de sus padres con enfermedad mental, el $47,8 \%$ tiene padres separados, un $42 \%$ en unión libre y $10,3 \%$ no vive con sus padres; el 57,6 \% se lleva mejor con la madre, el $17 \%$ con hermanos, $16,5 \%$ con el padre, $5,8 \%$ no se lleva bien con ninguno y el 3,1\% con todos. El Alfa de Cronbach fue de 0,867 para la escala de Autoestima de Rosenberg, y del 0,8 para Escala desesperanza Beck, ambas escalas presentan un grado de confiabilidad elevada.

El riesgo de suicidio o grado de desesperanza fue "ninguno o mínimo" (59,4\%), "leve” (26,8\%), "moderado" (12,9\%) y "alto" $(0,9 \%)$. El factor determinante del riesgo de suicidio fue el motivacional $(\bar{x}: 1,70)$, seguido por el afectivo $(\overline{\boldsymbol{X}}:, 88)$ y cognitivo $(\overline{\boldsymbol{X}}: 1,27)$. Las edades de mayor riesgo suicida fueron: 14 años (11,6 \% mínimo; 3,1 \% leve; 0,9 \% moderado), 15 (11,02\% mínimo; 8\% leve; 2,2\% moderado), 16 (23,7\% mínimo; $8 \%$ leve; 6,3\% moderado) y 17 años (7,6\% mínimo; 4,5\% leve; 3,6\% moderado). El grado $9^{\circ}$ presenta riesgo mínimo $(20,1 \%)$, leve $(14,3 \%)$, moderado $(1,3 \%)$ y alto $(0,9 \%)$. La edad de 16 años presenta riesgos en tres niveles: ninguno/mínimo $(23,7 \%)$, leve $(8,0 \%)$ y moderado $(6,3 \%)$. El riesgo alto $(0,9 \%)$ se presentó a los 15 años. La autoestima tuvo los siguientes valores: alta (21,9\%), media-normal $(76,8 \%)$ y baja $(1,3 \%)$. La autoestima es elevada en edades entre 13-15 años (36,6\%), y 16-19 años (54,5\%), en $9^{\circ}(6,3 \%)$ y en $10^{\circ}(8 \%)$ grado. En mujeres, el riesgo suicida fue mínimo $(33,5 \%)$ y alto $(0,9 \%)$, y en hombres mínimo $(29.5 \%)$, leve $(17 \%, 9 \%)$ y moderado $(7,10 \%)$. La autoestima fue baja $(1,30 \%)$, normal-moderada $(34,8 \%)$ y 
elevada $(12,9 \%)$ en mujeres, y en hombres fue baja $(0 \%)$; normal-moderada ( $42 \%)$ y alta $(8,9 \%)$. La autoestima baja se presentó en mujeres de 15 años y en $9^{\circ}$ grado $(0,90 \%)$ y con padres separados $(1,30 \%)$, fue elevada en hijos con uno o más hermanos $(79,09 \%)$, en quienes conviven con ambos padres $(40,20 \%)$ y se llevan mejor con su madre. Estudiantes que refieren sentirse tristes la mayor parte del día presentan niveles de autoestima baja $(70,7 \%)$, media-normal $(4,90 \%)$ y elevada $(24,40 \%)$.

El coeficiente de correlación utilizado fue el coeficiente de Pearson. Se hallaron correlaciones muy bajas entre autoestima baja con factor motivacional $(\mathrm{p}=, 198$; $\mathrm{s}=$
,003) y con el factor cognitivo ( $\mathrm{p}=, 192 ; \mathrm{s}=, 004)$. El auto concepto positivo tuvo correlaciones muy bajas con: la actitud positiva hacia sí mismo ( $\mathrm{p}=, 181 ; \mathrm{s}=, 007)$ y sentirse satisfecho consigo mismo $(\mathrm{p}=, 171 ; \mathrm{s}=, 010)$. El riesgo suicida fue directamente proporcional al factor motivacional $(\mathrm{p}=, 734 ; \mathrm{s}=, 000)$ "correlación alta, factor cognitivo $(\mathrm{p}=$ $, 595 ; \mathrm{s}=, 000)$ "correlación moderada", y correlaciones muy bajas con el hecho de pensar que se es "inútil" ( $\mathrm{p}=, 142$; $\mathrm{s}=$,033), no sentirse orgulloso e inclinación a pensar en el fracaso $(\mathrm{p}=, 181 ; \mathrm{s}=, 006)$, y de ser malas personas $(\mathrm{p}=$ $, 187 ; s=, 005)$. Quienes afirman no sentir respeto por sí mismos también generan pensamientos de inutilidad $(\mathrm{p}=$ ,302; $\mathrm{s}=, 000)$ (tabla 1) "correlación baja".

Tabla 1. Correlaciones Riesgo suicida RS (desesperanza), factores de riesgo y autoestima.

\begin{tabular}{|c|c|c|c|}
\hline Riesgo & Factor motivacional & Cognitivo & $\begin{array}{l}\text { Pensar que se es un } \\
\text { "inútil" }\end{array}$ \\
\hline Riesgo alto de desesperanza & $\begin{array}{c}(\mathrm{p}=, 734 ; \mathrm{s}=, 000) \\
\text { Correlación alta }\end{array}$ & $\begin{array}{c}(\mathrm{p}=, 595 ; \mathrm{s}=, 000) \\
\text { Correlación moderada }\end{array}$ & $\begin{array}{l}(\mathrm{p}=, 142 ; \mathrm{s}=, 033) \\
\text { Correlación muy baja }\end{array}$ \\
\hline Riesgo & Futuro vago e incierto & Idea de un futuro oscuro & $\begin{array}{c}\text { Ver el futuro de forma } \\
\text { desagradable }\end{array}$ \\
\hline Riesgo alto de desesperanza & $\begin{array}{c}(\mathrm{p}=, 460 ; \mathrm{s}=, 000) \\
\text { Correlación baja }\end{array}$ & $\begin{array}{c}(\mathrm{p}=, 640 ; \mathrm{s}=, 000) \\
\text { Correlación moderada }\end{array}$ & $\begin{array}{c}(\mathrm{p}=, 532 ; \mathrm{s}=, 000) \\
\text { Correlación moderada }\end{array}$ \\
\hline Riesgo & $\begin{array}{l}\text { Impotencia para } \\
\text { cambiar las cosas }\end{array}$ & $\begin{array}{l}\text { Darse por vencido, } \\
\text { renunciar }\end{array}$ & No conseguir lo deseado \\
\hline \multirow[t]{2}{*}{ Riesgo alto de desesperanza } & $\begin{array}{l}(\mathrm{p}=, 590 ; \mathrm{s}=, 000) \\
\text { Correlación moderada }\end{array}$ & $\begin{array}{c}(\mathrm{p}=, 403 ; \mathrm{s}=, 000) \\
\text { Correlación baja }\end{array}$ & $\begin{array}{c}(\mathrm{p}=, 474 ; \mathrm{s}=, 000) \\
\text { Correlación baja }\end{array}$ \\
\hline & Incredulidad & Pesimismo & \\
\hline Riesgo alto de desesperanza & $\begin{array}{c}(\mathrm{p}=, 412 ; \mathrm{s}=, 000) \\
\text { Correlación baja }\end{array}$ & $\begin{array}{c}(\mathrm{p}=, 476 ; \mathrm{s}=, 000) \\
\text { Correlación baja }\end{array}$ & - \\
\hline Riesgo & $\begin{array}{l}\text { Pensar que son } \\
\text { fracasados }\end{array}$ & $\begin{array}{c}\text { Pensar que son malas } \\
\text { personas }\end{array}$ & $\begin{array}{l}\text { Creerse inútil - sin } \\
\text { respeto por sí mismo }\end{array}$ \\
\hline $\begin{array}{l}\text { Creen no tener cosas para } \\
\text { sentirse orgullosos }\end{array}$ & $\begin{array}{c}(\mathrm{p}=, 181 ; \mathrm{s}=, 006) \\
\text { Correlación muy baja }\end{array}$ & $\begin{array}{l}(\mathrm{p}=, 187 ; \mathrm{s}=, 005) \\
\text { Correlación muy baja }\end{array}$ & $\begin{array}{l}(\mathrm{p}=, 302 ; \mathrm{s}=, 000) \\
\text { Correlación baja }\end{array}$ \\
\hline Riesgo & Factor motivacional & Factor cognitivo & \\
\hline Autoestima baja & $\begin{array}{l}(\mathrm{p}=, 198 ; \mathrm{s}=, 003) \\
\text { Correlación muy baja }\end{array}$ & $\begin{array}{c}(\mathrm{p}=, 192 ; \mathrm{s}=, 004) \\
\text { Correlación muy baja }\end{array}$ & - \\
\hline
\end{tabular}

Fuente: elaboración propia.

\section{DISCUSIÓN}

El fenómeno del suicidio es un evento de origen multicausal, lo que quiere decir que los hechos que intervienen en su ejecución suelen ser múltiples y no presentan un mismo origen. Al respecto Shneidman ${ }^{19}$ indica que en muchos parasuicidios y suicidios prima más, una reacción derivada de motivaciones a menudo poco racionalizadas por los sujetos, que la decisión concientizada frente a la situación estresora. En la 
población investigada el principal factor de riesgo suicida fue de tipo motivacional, seguido por el factor afectivo y cognitivo, de lo cual es posible afirmar que la desesperanza es impulsada principalmente por aspectos motivacionales de un fuerte contenido emocional-afectivo, más que por cogniciones sólidamente configuradas; de allí que la autoestima requiera un nivel importante de cognición, pero que se determine de acuerdo a la vivencia afectiva de cada sujeto, lo cual está íntimamente relacionado con la experiencia subjetiva de cada persona y su capacidad de sobreponerse y resistir ante la adversidad (resiliencia) ${ }^{20}$. De hecho, aquello que determina los niveles de desesperanza guarda relación con el autoconcepto creado en las relaciones afectivas con personas importantes en la vida, y los antecedentes depresivos a nivel personal y familiar, que actúan a modo de factores determinantes y predictores de la conducta suicida $^{34,6}$. Estos resultados son similares a los hallazgos de investigaciones que revelan el incremento del riesgo de suicidio en adolescentes cuando persisten problemas de motivación respecto al funcionamiento familiar, depresión, presiones ambientales, consumo de sustancias psicoactivas, fracasos escolares y otras variables socio ambientales ${ }^{10,32,35-38}$

En la población evaluada el riesgo de suicido no tuvo correlación significativa con la autoestima baja, aunque sí con la desmotivación individual que responde a estados psicológicos alterados y también, a toda ambivalencia afectiva que afecte la autoestima ${ }^{14}$. Esta desmotivación desde una mirada psicológica puede asociarse a problemas en uno o varios de dominios del Self (interpersonal, familiar, grupal, prospectivo, organizacional), puesto que torna a los adolescentes proclives a presentar problemas de ajuste ante demandas de su entorno, elevando la posibilidad de experimentar mayores riesgos psicosociales. Ergo debilitan su autoestima, se aíslan y son menos asertivos, aun cuando operen adecuadamente en otros contextos ${ }^{14,24,32}$. Asimismo cuando las habilidades para la vida no trabajan adecuadamente y el adolescente no logra resolver los conflictos emergentes, éste puede desmotivarse y quedar a expensas de temores, ideas de muerte y expectativas frustradas ${ }^{24}$, aspecto que fue análogo a lo encontrado en la investigación puesto que, los factores motivaciones y cognitivos que los determinan fueron determinantes en el riesgo suicida. Los adolescentes evaluados con riesgo suicida revelan ser castigados pos sus padres injustamente, y el sentirse triste la mayor parte del día. Dichos factores coinciden con los factores de riesgo elevado encontrados en otras investigaciones ${ }^{16,9,22}$, y son fuente de dilemas sociales y de crisis. Así, mientras para algunos la crisis se vive como la suma de momentos catastróficos, para otros puede constituir un evento de permanente riesgo y preocupación ${ }^{26,38-39}$.

La investigación encontró que el riesgo suicida grave/ elevado es mayor en mujeres, mientras el riesgo leve y moderado es mayor en hombres, lo cual disiente de estudios que revelan una prevalencia masculina ${ }^{35,37,40}$, aun cuando en la actualidad las tasas sugieren un curso equivalente ${ }^{37,38,41-43}$, especialmente cuando se relacionan al consumo de alcohol y otras sustancias psicoactivas ${ }^{44}$. Cuando se trata género los intentos de suicidio pueden ser mayores en mujeres adolescentes, al ser más coaccionadas por sus padres o por imposiciones derivadas de la cultura patriarcal1. Asimismo, el estudio nacional sobre salud mental en Colombia reveló que la mujer adolescente es más vulnerable en su salud mental que el hombre, ya que suelen ser mayormente sensibles a trastornos fóbicos (fobia social), la depresión y la bipolaridad ${ }^{10,32,40}$

Hubo correlación entre riesgo suicida leve, edad (16-18 años), y autoestima baja (leve). El incremento de dichos aspectos eleva el riesgo de suicidio en las edades 13-15 años, y 15-17 años, lo cual es consistente a estudios que revelan dichas edades como de mayor riesgo en la adolescencia $^{37,41,45}$, misma que está asociada a un elevado nivel de confusión emocional, cambios físicos, variaciones de autoestima y fluctuaciones afectivas significativas respecto a normas, proyectos de vida e influencia del grupo de pares ${ }^{26,24}$. En contraste, una proyección futura y un autoconcepto positivo pueden protegerlos de quitarse la vida1. Sin embargo cuando los factores protectores son escasos o débiles, la desesperanza puede convertirse en el centro regulador de la vida emocional ${ }^{34,35,17}$. En la población investigada dicho aspecto se asoció al hecho que la desesperanza aparezca como respuesta disfuncional a la dificultad de examinar cognitivamente situaciones complicadas, además de expectativas negativas, ideas de tener un futuro oscuro de escaso crecimiento-apoyo, además, de la sensación de impotencia derivada de no lograr superar sus problemas. Ergo, ver el futuro de forma áspera o complicada eleva los riesgos de suicidio, depresión y problemas de auto-ajuste social ${ }^{35}$.

Otros factores encontrados fueron: la idea que su futuro es vago/incierto, dificultad para satisfacer necesidades, incredulidad ante el apoyo de otros, y pesimismo ante cosas que anhelan; estos elementos se constituyen en predictores del riesgo suicida ${ }^{43}$. A esto debe agregarse: disfuncionalidad familiar, conflictividad social, vivir una 
adolescencia conflictiva, trastornos del humor y consumo de sustancias psicoactivas ${ }^{30,44}$. Para los adolescentes las relaciones parentales y con sus pares constituyen el sustrato de donde emergen frecuentemente conflictos, ya que en ellos pueden generarse múltiples problemas y factores de riesgo. Estudios demuestran que al menos tres de cada 10 estudiantes han presentado una conducta suicida durante su vida y de ellos el $8 \%$ llevo a cabo un intento de suicidio10. En Colombia, el comportamiento suicida durante la última década no ha presentado una variación significativa en las tasas, pero se evidencia que las poblaciones principalmente afectadas son adolescentes y jóvenes hombres, mujeres y adultos mayores ${ }^{3,17,18,45}$.

La autoestima fue alta en los adolescentes aun cuando presentaron algún tipo de riesgo suicida, lo que revela que la autoestima no es un elemento determinante del riesgo suicida, aspecto compartido por otras investigaciones ${ }^{1,26,27,45}$. Caso contrario sucede con el ítem: "sentirse triste la mayor parte del día durante el último mes", pues quienes lo presentan cuentan con un mayor grado de desesperanza y pueden presentar problemas de autoestima, lo cual es similar a los encontrado por diversos estudios ${ }^{34,45,46}$. La autoestima se constituye con base en la actitud del adolescente ante los diferentes contextos, por lo que éste puede reaccionar de diversas maneras de acuerdo a la forma como interpreta las interacciones con pares y adultos, de allí se consolidan el Self y la robustez emocional ${ }^{14}$. El suicidio presenta una condición multicausal y por ello no puede reducirse a una sola causa o efecto; en consecuencia, puede considerarse como un fenómeno complejo y multidimensiona ${ }^{11,39}$.

\section{CONCLUSIÓN}

A nivel de relaciones intrafamiliares $3 / 10$ estudiantes se lleva bien con todos en su familia, pero con quien mejor conviven y a la vez discuten es con la madre. $3 / 10$ estudiantes afirmó tener tristeza todo el día durante el último mes, y fueron quienes presentaron riesgo suicida moderado y alto, lo cual muestra una relación importante entre depresión e ideación suicida. 4/10 estudiantes presenta riesgo leve, moderado o alto; $6 / 10$ riesgo mínimo. El riesgo de suicidio o grado de desesperanza no se debe específicamente a la baja autoestima, no obstante, la relación no debe pasarse por alto, pues requiere ser comprendida en asociación relacional con otros factores precipitantes. El suicidio es un fenómeno complejomultidimensional que afecta la salud de personas, familias, grupos y comunidades, deteriora su calidad, expectativa de vida y disminuye los años productivos.
El riesgo suicida tiene su asiento más en factores motivacionales y cognitivos que en la autoestima baja. La edad de mayor riesgo suicida en ambos géneros suicida fue entre 14-17 años, y que se incrementa en mujeres posiblemente a razón de pautas de crianza machistas, que suprimen la posibilidad de individualidad de la mujer. Aunque esta hipótesis no se encuentra comprobada en ésta investigación, se recomienda indagarla en próximos estudios, tomando en cuenta la violencia de género y la orientación patriarcal de muchas pautas de crianza. Se encontró que el riesgo suicida relaciones es común en quienes se encuentran entre 14-17 años, están tristes la mayor parte del día, tienen padres separados, cursan $9^{\circ}$ grado, y su autoestima es normal o baja.

Los factores motivacionales y cognitivos generan dilemas existenciales que apuntalan la ideación suicida y elevan los riesgos de suicidio en los adolescentes. La desesperanza se asocia al hecho de pensar que se es un inútil, y creer que no se cuenta con cualidades para sentirse orgulloso, lo que puede inclinar al adolescente a pensar en el fracaso y en ser una mala persona. Aquellas personas con pensamientos de inutilidad, que se rinden a las dificultades o renuncian a sus objetivos tienen un riesgo alto de desesperanza.

El riesgo alto de desesperanza se asoció también a tener un futuro oscuro, desagradable, vago e incierto (factor cognitivo), e impotencia para cambiar las cosas, pesimismo, frustración, incredulidad ante otros, y un elevado desilusión (factor motivacional). La autoestima decrece entre los 13 y los 15 años, siendo baja en grado noveno al verse influenciada por la separación de los padres y los conflictos con la figura materna. El elevado auto-concepto está directamente correlacionado con una actitud positiva hacia sí mismo.

El riesgo suicida se relaciona con: interacciones intrafamiliares, problemas y dilemas propios de la adolescencia, así como también, a un mal auto-concepto, débil proyecto de vida. La autoestima normal y elevada es un factor protector ante la intensidad de los problemas socio-familiares y el incremento de diversos factores de riesgo psicosocial.

\section{DECLARACIÓN SOBRE CONFLICTO DE INTERESES}

Declaramos no tener ningún tipo de asociación comercial ni personal que suponga un conflicto de intereses en relación al artículo remitido. 


\section{REFERENCIAS BIBLIOGRÁFICAS}

1. Andrade JA. Aspectos psicosociales del comportamiento suicida en adolescentes. Itztacala [revista en la Internet]. 2012. [citado 2015 Nov 24]; 15(2): 688-721. Disponible en: http://www.revistas.unam.mx/index.php/repi/ article/view/32373/29770

2. Instituto Nacional de Medicina legal y Ciencias Forenses. Comportamiento del suicidio, Colombia; 2013 [Internet]. Bogotá: FORENSIS [citado 2015 Nov 24]. Disponible en: http://www.medicinalegal.gov. co/documents/10180/188820/FORENSIS + 2013 + 3+ suicidio.pdf/65a683b4-38b2-46a4-b32a-f2a0884b25bf

3. Instituto Nacional de Medicina legal y Ciencias Forenses. Comportamiento del suicidio, Colombia; 2012 [Internet]. Bogotá: FORENSIS [citado 2015 Nov 24]. Disponible en: http://www.medicinalegal.gov.co/documents/10180/ $34861 / 6+5$ + suicidio + forensis + 2012.pdf/654be51860d0-4979-b899-f26c83d1bb8c

4. Instituto Nacional de Medicina legal y Ciencias Forenses. Comportamiento del suicidio, Colombia; 2014 [Internet]. Bogotá: FORENSIS [citado 2015 Nov 24]. Disponible en: http://www. medicinalegal.gov.co/documents/88730/1656998/ Forensis + Interactivo + 2014.24-JULpdf.pdf/9085ad79d2a9-4c0d-a17b-f845ab96534b

5. Bellaa ME, Fernández R, Willington JM. Intento de suicidio en niños y adolescentes: depresión y trastorno de conducta disocial como patologías más frecuentes. Arch Argent Pediatr [revista en la Internet]. 2010 [citado 2015 Nov 24]; 108(2):124-29. Disponible en http://www2.sap.org.ar/docs/publicaciones/ archivosarg/2010/v108n2a06.pdf

6. Villalobos-Galvis FH. Situación de la conducta suicida en estudiantes de colegios y universidades de San Juan de Pasto, Colombia. Salud Ment [revista en la Internet]. 2009 Abr [citado 2016 Mayo 31]; 32(2): 165-71. Disponible en: http://www.scielo.org.mx/scielo.php?script = sci arttext\&pid $=$ S0185-33252009000200009\&lng $=$ es

7. Pérez-Barrero SA. El suicidio, comportamiento y prevención. Rev Cubana Med Gen Integr [Internet]. 1999 Abr [citado 2016 Mayo 31]; 15(2): 196-217. Disponible en: http://scielo.sld.cu/scielo.php? script $=$ sci arttext\&pid $=$ S0864-21251999000200013\&lng = es

8. Palacio A. La comprensión clásica del suicidio de Emile Durkheim en nuestros días. Affectio Societatis. 2010 Junio [citado 2016 Mayo 31]; 7(12): 1-13. Disponible en http://aprendeenlinea.udea.edu.co/revistas/index.php/ affectiosocietatis/article/viewFile/6318/6520

9. Valdivia M, Ebner D, Fierro V, Gajardo C, Miranda R. Hospitalización por intento de suicidio en población pediátrica: una revisión de cuatro años. Rev. chil. neuro-psiquiatr. [Internet]. 2001 Sep [citado 2016 Mayo 31]; 39(3): 211-18. Disponible en: http://www.scielo.cl/ scielo.php?pid $=$ S0717-92272001000300005\&script $=$ sci arttext
10. López E, Musitu G, Murgui S, Moreno A. Clima familiar, clima escolar y satisfacción con la vida en adolescentes. Revista Mexicana de Psicología. 2013; 25(1):119-28.

11. Mardomingo MJ, Catolin Zamora ML. Intentos de suicidio en la infancia y adolescencia: características epidemiológicas. An Esp Pediatr 1992; 37(1): 29-32

12. Lester $D$. The effectiveness of suicide prevention centers: a review. Suicide Life Threat Behavior. 1997; 27(3): 304-10.

13. Carvajal G, Caro CV. ideación suicida en la adolescencia: una explicación de tres de sus variables asociadas en Bogotá, 2009. Colomb. Medicina. [Internet]. 2011 Junio [citado el 31 de 2016 podrá]; 42 (2 Suppl 1): 35-44. Disponible en: http://www.scielo. org.co $/$ scielo.php? script $=$ sci_arttext\&pid $=$ S1657 95342011000500006\&lng = en .

14. Estévez E, Murgui S, Moreno, D, Musitu, G. Estilos de comunicación familiar, actitud hacia la autoridad institucional y conducta violenta del adolescente en la escuela. 2007; 19(1): 108-113. Disponible en http:// www.psicothema.com/pdf/3335.pdf

15. Sarro B. De la Cruz C. Los suicidios. Primera edición. España: Editorial Martínez Roca; 1991.

16. Chávez-Hernández AM, Leenaars AA. Edwin S Shneidman y la suicidología moderna. Salud Ment [revista en la Internet]. 2010 Ago [citado 2016 Mayo 31]; 33(4): 355-360. Disponible en: http://www.scielo. org. $\mathrm{mx} /$ scielo. php ? script $=$ sci_arttext\&pid $=$ S0185$33252010000400008 \& \ln$ = es.

17. Cifuentes SL. Violencia autoinfligida desde el sistema médico legal colombiano. En: Forensis, datos para la vida. Instituto Nacional de Medicina Legal y Ciencias Forenses. Bogotá; 2013.

18. Instituto Nacional de Medicina legal y Ciencias Forenses. Quitarse la vida cuando ésta aún comienza. Boletín epidemiológico trimestral. [Internet]. 2013 Diciembre [citado 2016 Mayo 31;] 6(8): 1-14. Disponible en ttp:// www.medicinalegal.gov.co/documents/10180/28521 $/ 2+$ suicidios-jovenes.pdf/136d4b84-821a-430f-befc57b72b17fb6d

19. Shneidman ES. A possible classification of suicidal acts Based on Murray's need system. Suicide Life-threatening Behavior. 1980;10(3):175-81

20. Gutiérrez M, Romero I. Resiliencia, bienestar subjetivo y actitudes de los adolescentes hacia el consumo de drogas en Angola. Anal. Psicol. [Internet]. 2014 Mayo [citado 2016 Mayo 31]; 30(2): 608-19. Disponible en: http://scielo.isciii.es/scielo.php?script = sci_arttext\&pi $\mathrm{d}=$ S0212-97282014000200024

21. Durkheim E. El suicidio. Primera edición. Argentina: Editorial Schapire; 1965.

22. Silva Dl, Vicente B, Saldivia S, Kohn R. Conducta suicida y trastornos psiquiátricos en Chile, un estudio poblacional. Rev. méd. Chile [Internet]. 2013 Oct [citado 2016 Mayo 31]; 141(10): 1275-1282. Disponible en: http://www.scielo.cl/scielo.php? script = sci arttext\&pid $=$ S0034-98872013001000006\&lng = es 
23. Mack JE. Suicide and depression among adolescent and young adults. Adolescent suicide: aix arquitectural model, Ed. Klerman GL, editor. Washington: American Psychiatryc Press; 1986

24. Musitu G, Cava MJ. El rol del apoyo social en el ajuste de los adolescentes. Intervención Psicosocial. 2003; 12(2): 179-192.

25. Organización Mundial de la Salud. Suicidio [Internet]. OMS [citado 2015 Nov 24] Disponible en: http://www. who.int/publications/es/

26. Aberastury A, Knobel F. La adolescencia normal. Un enfoque psicoanalítico. México: Paidós; 2004.

27. Atienza, FL, Moreno Y, Balaguer I. Análisis de la dimensionalidad de la Escala de Autoestima de Rosenberg en una muestra de adolescentes valencianos. Revista de Psicología Universitas Tarraconensis. 2000;32(2): 29-42.

28. Rojas-Barahona C, Zegers B, Förster, C. La escala de autoestima de Rosenberg: Validación para Chile en una muestra de jóvenes adultos, adultos y adultos mayores. Rev. méd. Chile [Internet]. 2009 Jun [citado 2016 Jun 01]; 137(6): 791-800. Disponible en:http://www.scielo. cl/scielo.php?pid $=$ S003498872009000600009\&script $=$ sci_arttext

29. Serot NM, Teevan RC. Perception of the Parent Child Relationship and Its Relation to Child Development. Child Development. 1961; 32(2): 373-8.

30. Baumrind D. The influence of parenting style on adolescent competence and substance use. Journal of Early Adolescence. 1991; 11(1): 56-95.

31. Rosenberg M. Society and The adolescent self-image. Princeton: Princeton University Press; 1965.

32. Cañón SC. Factores de riesgo asociados a conductas suicidas en niños y adolescentes. Arch Med. 2011; 11(1): $62-7$

33. Hernández SR, Fernández CC, Baptista LP. Fundamentos de metodología de la investigación. 4ta Edición. México: McGraw Hill; 2006

34. Beck AT, Steer RA, Beck JS, Newman CF. Hopelessness, depression, suicidal ideation, and clinical diagnosis of depression. Suicide and Life-Threatening Behavior. 1993; 23(2): 139-45.

35. Beck AT, Weissman A, Lester D, Trexler L. The measurement of pessimism: The Hopelessness Scale.
Journal of Consulting and Clinical Psychology. 1974; 42(6): 861-5.

36. Toro G, Paniagua S, González P, Montoya GB. Caracterización de adolescentes escolarizados con riesgo de suicidio, Medellín, 2006. Revista de la Facultad Nacional de Salud Pública. 2006; 27(3): 302-308.

37. Pérez I, Téllez D, Ibáñez M. Caracterización de factores asociados con comportamiento suicida en adolescentes estudiantes de octavo grado de 3 colegios en Bogotá. Revista Colombiana de Psiquiatría. 2012; 41(1):26-47.

38. Villalobos B, Crespo J. Intentos de suicidio en niños y adolescentes en la consulta de emergencia del Hospital Miguel Pérez Carreño: Junio 2002-Mayo 2003. Archivos Venezolanos de Psiquiatría y Neurología. 2004; 50(103):6-12.

39. Rubenowitz E, Waern M, Wilelmson K, Allbeck P. Life events and psychosocial factors in elderly suicidescase-control study. Psychol Med. 2001;31(1):1193-202.

40. Echeburúa E. Las múltiples caras del suicidio en la clínica psicológica. Terapia psicológica [Internet]. 2015 Jul [citado 2016 Jun 01]; 33(2): 117-26. Disponible en: http://www.scielo.cl/scielo.php?script = sci_arttext\&pi $\mathrm{d}=\mathrm{S} 0718-48082015000200006$

41. Martínez AB, Guinsberg E. Investigación Cualitativa al estudio del intento de suicidio en Jóvenes de Tabasco. Rev. Fac. Nac. Salud Pública 2009; 27(1): 32-38

42. Martínez-Rozo A, Uribe-Rodríguez A, VelázquezGonzález H. La discapacidad y su estado actual en la legislación colombiana. Duazary. 2015 Jun; 12(1):49-58.

43. McMillan D, Gilbody S, Beresford E, Neilly L. Can we predict suicide and non-fatal self-harm with the Beck Hopelessness Scale? A meta-analysis. Psychological Medicine. 2007; 37(6): 769-78.

44. Mondragón L, Saltijeral MT, Bimbela A, Borges G. La ideación suicida y se relación con la desesperanza, el abuso de drogas y alcohol. Salud Mental. 1998; 21(5): 20-7.

45. Gonzales-Portillo J, Gil-Arévalo J, Hernández-Botero D, Henao-Sánchez L. Evaluación de las expectativas negativas y tipo de riesgo suicida en estudiantes de $9^{\circ}$, $10^{\circ}$ y $11^{\circ}$ de una Institución Educativa del Departamento del Quindío. Duazary. 2016 enero; 13 (1): 7-14

46. Rodríguez-Díaz A, Mejía-Moreu Y. Bullying: un fenómeno por transformar. Duazary. 2012; 9(1):98-104.

Para citar este artículo: Andrade-Salazar J, Pretel L, Ortega-Maya P, Ramirez-Avilés E, Carvajal-Valencia J. Autoestima y desesperanza en adolescentes de una institución educativa del Quindío. Duazary. 2017 julio; 14 (2): 179 - 187. Doi: http://dx.doi. org/10.21676/2389783X.1968 